\title{
Born this Way?
}

\section{Neurowissenschaftliche Anthropologien zwischen \\ Determinismus und Veränderbarkeit}

Laura-Christin Krannich

\section{Ein neues Menschenbild?}

Was ist der Mensch? Eine Frage, die schon tausendfach beantwortet wurde und immer wieder neu nach Beantwortung verlangt. Einen wichtigen Beitrag in gesellschaftlich geführten Debatten um den Menschen erbringen gegenwärtig insbesondere die Neurowissenschaften, ${ }^{1}$ denen in dieser Angelegenheit ein hohes Erklärungspotenzial zugetraut wird. ${ }^{2}$ Denn sie liefern hard facts, können ihre Thesen mittels umfangreicher Datensätze und bildgebender Verfahren untermauern. So scheinen sie klare Antworten auf uralte Fragen zu liefern: Wie ist der Mensch denn nun wirklich?

Eben diese Frage trieb auch den prominenten deutschen Neurowissenschaftler Gerhard Roth um, der die Klärung zunächst klassisch über ein Philosophiestudium suchte:

»Ich hatte mich entschlossen, Philosophie zu studieren, um zu erfahren, wie Wahrnehmung funktioniert und wie Erkenntnis und Wissen zustande kommen, was Geist ist und wie man wahre Aussagen von falschen unterscheidet. In vielen Vorlesungen erfuhr ich, was Platon, Aristoteles, Descartes, Leibniz, Kant und Hegel zu diesen Fragen gesagt hatten. Die Frage allerdings, die mich brennend interessiert, nämlich 'wer hat womit recht?,

\footnotetext{
1 "Neurowissenschaften" sind natürlich ein weites Feld. Ich beziehe mich hier auf die kognitive Neurowissenschaft, die auf die Erforschung des menschlichen Gehirns und dabei genauer auf komplexe Phänomene des Denkens (z.B. Aufmerksamkeit, Objekterkennung, Sprache) spezialisiert ist, also auf das, was in der Regel als »Hirnforschung« bezeichnet wird.

2 So erklärte Gerhard Roth in der Wirtschaftswoche, dass politische Entscheidungen keineswegs so rational getroffen werden wie es uns lieb wäre, und rät Politiker/-innen zu einer Runde Schlaf, wenn Entscheidungen "alternativlos" erscheinen; Ferdinand Knauss, Wer sich nicht entscheiden kann, sollte schlafen gehen, WirtschaftsWoche (31.01.2014), URL: https://www.wiwo.de/erfolg/trends/hirnforscher-gerhardroth-erst-schlafen-dann-entscheiden/9415864-3.html (17.01.2019).
} 
wurde nicht behandelt. Wenn ich sie in den Seminaren stellte, wurde ich belächelt; diese Frage galt als unpassend. Hierin steckte - wie ich erst viel später erkannte - ein gutes Stück Ehrlichkeit. Wie hätte man die Frage 'Wer hat recht?` auch beantworten können! «

Die Frustration angesichts der Frage "Wie ist es nun wirklich?« teilt Roth sicher mit einer grossen Menge von Studierenden, die sich ebenfalls mehr vom Studium erhofft haben als Dozent/-innen, die ihnen tadelnd zurufen: »Da muss man differenzieren!« Roth jedenfalls gab sich nicht zufrieden und suchte nach erfolgter Promotion in der Philosophie schliesslich in der Biologie nach Antworten. Und siehe da: Der Biologe Gerhard Roth kann von der "Lösung der Frage, wie Wahrnehmen und Denken zustande kommen und in welchem Verhältnis Geist und Gehirn zueinander stehen «", sprechen. Mit der neurowissenschaftlichen Forschung verbindet sich also der Anspruch, grosse Menschheitsfragen nicht nur immer wieder neu zu durchdenken, sondern tatsächlich lösen zu können.

Dieser erkenntnistheoretische Optimismus verband sich oft genug mit steilen Thesen zur Willensfreiheit des Menschen. So formuliert Wolf Singer, ein weiterer der breiteren Öffentlichkeit bekannter Neurowissenschaftler:

"Die Annahme [...], wir seien voll verantwortlich für das, was wir tun, weil wir es ja auch anders hätten machen können, ist aus neurobiologischer Perspektive nicht haltbar. Neuronale Prozesse sind deterministisch. Gibt man der nichtsprachlichen Hirnhälfte einen Befehl, führt die Person diesen aus, ohne sich derVerursachung bewusst zu werden. Fragt man dann nach dem Grund für die Aktion, erhält man eine vernünftige Begründung, die aber mit der eigentlichen Ursache nichts zu tun hat. $\|^{5}$

In ähnlicher Weise prognostiziert das 2004 publizierte "Manifest» zu Gegenwart und Zukunft der Hirnforschung, dass die Ergebnisse neurowissenschaftlicher Forschung zu »einer Veränderung unseres Menschenbildes führen ${ }^{6}{ }^{6}$ werden. Öffentlichkeitswirksam wurden

\footnotetext{
3 Gerhard Roth, Das Gehirn und seine Wirklichkeit. Kognitive Neurobiologie und ihre philosophischen Konsequenzen, Frankfurt a.M. 1997, 11.

4 Ebd., 12 (Hervorhebung Vf.).

5 Wolf Singer, Ein neues Menschenbild. Gespräche über Hirnforschung, Frankfurt a.M. 2003, 20.

6 Christian E. Elgar u.a., Das Manifest. Elf führende Neurowissenschaftler über Gegenwart und Zukunft der Hirnforschung, in: Geist\&Gehirn 6 (2003), 30-37, hier
} 
auch Forderungen zur Veränderung des Strafrechts auf Basis dieser Erkenntnisse. $^{7}$

Diese Annahme weitreichender Konsequenzen neurowissenschaftlicher Erkenntnisse für alle Lebensbereiche hat dazu geführt, dass Neurowissenschaften teils als Leitwissenschaft des noch recht jungen 21. Jahrhunderts beschrieben worden sind, als Disziplin also, deren Forschungsergebnisse und -methoden für andere Wissenschaftsbereiche erhebliches Innovationspotenzial beinhalten - und: die sich selbst als eine solche Wissenschaft sieht und von aussen derart anerkannt wird. ${ }^{8}$ Zweifelsohne finden sich eine ganze Reihe von Vertreter/-innen der Neurowissenschaften, die diesen Anspruch erheben, und ebenso unzweifelhaft wird den Neurowissenschaften dieses Potenzial oftmals zugesprochen.

Doch das ist nur ein Teil der Wahrheit. Denn zum einen wird der Erklärungsanspruch der Neurowissenschaften intradisziplinär durchaus unterschiedlich (und nicht selten: zurückhaltender als oben geschildert) beurteilt. ${ }^{9}$ Und zum anderen ist nach dem Optimismus der 2000er Jahre zunehmend Ernüchterung zu beobachten. Zehn Jahre nach der Veröffentlichung des erwähnten "Manifests" werden die damals aufgestellten Prognosen und Ansprüche in einer von fünfzehn Neurowissenschaftler/-innen erarbeiteten Bilanzierung als überzogen beurteilt. Das Vorgenommene wurde nicht erreicht, mehr noch: Die damals gesetzten Ziele erscheinen im Rückblick in wissenschaftstheoretischer und methodischer Hinsicht problematisch. Die Forschenden sprechen sich angesichts dieser Ergebnisse für eine stärkere interdisziplinäre Vernetzung, insbesondere auch mit Philosophie und Sozialforschung, aus. ${ }^{10}$

Es lässt sich daraus folgern, dass die Natur des Menschen in den Neurowissenschaften möglicherweise viel weniger klar vor Augen steht und viel weniger hermetisch gefasst ist, als der Blick auf ei-

37; online verfügbar unter URL: https://www.spektrum.de/magazin/das-manifest/839085 (17.01.2019).

7 Michael Pauen/Gerhard Roth, Freiheit, Schuld und Verantwortung. Grundzüge einer naturalistischen Theorie der Willensfreiheit, Frankfurt a.M. 2008.

8 Norbert Herschkowitz, Möglichkeit und Grenzen der Neurowissenschaften: Ist die Neurowissenschaft eine Leitwissenschaft?, in: Aktualität und Vergänglichkeit der Leitwissenschaften, hg. v. Peter Rusterholz/Ruth Meyer Schweizer/Sara Margarita Zwahlen, Bern 2009, 131-142, hier 131.

9 Thorsten Heinemann, Populäre Wissenschaft. Hirnforschung zwischen Labor und Talkshow, Göttingen 2012, 157-160.

${ }^{10}$ Felix Tretter u.a., Memorandum »Reflexive Neurowissenschaft», in: Psychologie heute 3 (2014).Vgl. auch Josef Quitterer, Anspruch und Wirklichkeit. 10 Jahre nach dem Manifest der Hirnforscher, in: Herder Korrespondenz 69 (2015), 593-596. 
nige bekannte Konzeptionen nahelegen mag. Diesem Gedanken nachspürend, begebe ich mich auf einen kurzen Streifzug durch zwei Bereiche der neurowissenschaftlichen Forschung, die in ganz unterschiedlicher Weise um die Frage nach der Natur des Menschen kreisen: Im Kontext neurowissenschaftlicher Erforschung von Transsexualität begegnet die Frage, wann und in welcher Weise sich menschliche Geschlechtsidentität ausbildet und welche Rolle neurologische Strukturen dabei spielen: Wo, wann und wie bildet sich die menschliche Natur heraus? Ausserdem erfolgt ein kurzer Blick in neuroethische Debatten hinsichtlich der Möglichkeiten und Grenzen von nicht medizinisch-indizierten Eingriffen in das menschliche Gehirn (»Hirndoping «). Diskutiert wird hier, inwieweit Konzeptionen der menschlichen Natur als Kriterium für die Zulässigkeit oder Unzulässigkeit von Neuroenhancement dienen können. ${ }^{11}$

\section{Die Natur des Menschen in neurowissenschaftlichen Debatten}

\subsection{Transsexualität}

Transsexualität bezeichnet das Auseinandertreten des bei der Geburt zugeschriebenen, phänotypisch-körperlichen Geschlechts und des geschlechtlichen Selbsterlebens, das oftmals (aber keineswegs immer)

\footnotetext{
${ }^{11}$ Ich lasse damit bewusst die deutlich prominenteren Beispiele aussen vor. Die Infragestellung der Willensfreiheit in den Neurowissenschaften ist bereits umfangreich bearbeitet worden, vgl. z.B. Wolfgang Achtner, Willensfreiheit in Theologie und Neurowissenschaften. Ein historisch-systematischer Wegweiser, Darmstadt 2010; Andreas Klein, Ich bin so frei. Willensfreiheit in der philosophischen, neurobiologischen und theologischen Diskussion, Neukirchen-Vluyn 2012; Markus Mühling, Willensgebundenheit und notwendige Kontingenz als Bedingungen von Willensfreiheitserfahrungen, in: NZSTh 55 (2013), 161-187; Geran F. Dodson, Free Will, Neuroethics, Psychology and Theology, Wilmington 2017. Zur Erforschung von Religiosität, die teils unter dem unglücklich gewählten Begriff 'Neurotheologie‘ gefasst wird, vgl. Andrew Newberg/Eugene d'Aquili/Vince Rouse, Why God Won't Go Away. Brain Science and the Biology of Belief, New York 2001 (dt. Ausgabe: Der gedachte Gott. Wie Glaube im Gehirn entsteht, München 2003); Michael Blume, Neurotheologie. Hirnforscher erkunden den Glauben, Marburg 2009; Patrick McNamara, The Neuroscience of Religious Experience, Cambridge 2009. Zur Verhältnisbestimmung von Neurowissenschaften und Theologie vgl. Dirk Evers, Hirnforschung und Theologie, in: ThLZ 131 (2006) 11,1107-1122; Christina Aus der Au, Im Horizont der Anrede. Das theologische Menschenbild und seine Herausforderung durch die Neurowissenschaften, Göttingen 2011; Christian Ammer/Andreas Lindemann (Hg.), Hirnforschung und Menschenbild, Leipzig 2012.
} 
mit dem Wunsch nach Angleichung von Körper und Lebensweise an das Geschlechtserleben einhergeht. In die Ursachenforschung zur Aufklärung dieses Phänomens haben sich auch die Neurowissenschaften eingeschaltet. Sie gehen dabei von statistisch messbaren geschlechtsspezifischen Grössenunterschieden in menschlichen Gehirnen aus: In weiblichen Gehirnen ist das corpus callorum (auch als "Balken« bekannt) gegenüber männlichen Gehirnen verhältnismässig grösser, was zu einer intensiveren Vernetzung der rechten und linken Hirnhälfte führt. Dagegen sind zwei Intestinentalkerne des Hypothalamus (insb. INAH-3) in männlichen Gehirnen deutlich grösser als bei weiblichen. ${ }^{12}$ Diese Unterschiede bilden sich im Rahmen der embryonalen Entwicklung infolge von durch das Enzym Aromatase gesteuerten hormonellen Umwandlungsprozessen heraus, die zeitlich nach der körperlichen Ausprägung von Geschlechtsmerkmalen liegen. Kommt es nun bei diesen Ausbildungsprozessen zu Störungen, etwa Hemmung des Enzyms Aromatase, kann es passieren, dass die neuronale Geschlechtsentwicklung anders verläuft als die übrige körperliche Entwicklung. Transsexualität wird in diesem Sinne als Inkongruenz des Gehirn- und Körpergeschlechts verstanden, das auf den zeitlichen Abstand der Ausbildung des körperlich-genitalen und neuronalen Geschlechts zurückzuführen ist. ${ }^{13}$ Es wurde bereits vorgeschlagen, die Messung des corpus callorum zur Diagnostik von Transsexualität einzusetzen. ${ }^{14}$

Aus der neurologischen Struktur folgt die geschlechtliche Identität - diese Deutung verwundert nicht, auch wenn sie natürlich alles andere als unumstritten ist. Interessant für die Zwecke dieses Beitrags sind aber weniger Fragen nach der Tragfähigkeit von neurobiologischen Aussagen über Geschlechtlichkeit als vielmehr die Beobachtung, dass Geschlechtlichkeit hier als eine angeborene und unveränderliche Tatsache der menschlichen Natur betrachtet wird. Der entscheidende Ort der Herausbildung der menschlichen Natur ist dabei nicht die sexuelle Rekombination des Genoms, sondern die embryonale Entwicklung im Mutterleib, die zur Ausbildung der neuronalen Strukturen führt. Hier sind nun graduelle Unter-

\footnotetext{
${ }^{12}$ Mark Solms, The Biological Foundations of Gender: a Delicate Balance, in:Transsexualität in Theologie und Neurowissenschaften. Ergebnisse, Kontroversen, Perspektiven, hg. v. Gerhard Schreiber, Berlin/Boston 2016, 5-21, hier 12-15.

${ }^{13}$ Ebd., 15-16.Vgl. Dick F. Swaab/Laura Castellanos-Cruz/Ai-Min Bao, The Human Brain and Gender. Sexual Differentiation of Our Brains, in:Transsexualität in Theologie und Neurowissenschaften (s. Anm. 12), 23-41, hier 37-40.

${ }_{14}$ Yasunaro Yokota/Y. Kawamura/Y. Kameya, Callosal Shapes at the Midsagittal Plane. MRI Differences of Normal Males, Normal Females, and GID, in: Conf Proc IEEE Eng Med Biol Soc. 3 (2005), 3055-3058.
} 
schiede zu beobachten: Dick F. Swaab, Laura Castellanos-Cruz und Ai-Min Bao gehen davon aus, dass es hinsichtlich der Ausbildung von geschlechtlicher Identität keine nachgeburtlichen - insbesondere: keine sozialen - Faktoren gibt: "gender identity and sexual orientation arise in the womb ${ }^{15}$ Mark Solms dagegen bezeichnet die neuronalen Unterschiede in der Neuroanatomie als "minuscue" und hebt stärker die komplexe Verzahnung von Genexpression und Umwelt für die individuelle Merkmalsausprägung hervor; allerdings spricht er den Unterschieden in der Ausgangslage dabei eine wichtige Rolle zu, denn soziale Einflüsse seien nicht unabhängig von diesen initialen Unterschieden.Vielmehr wirkten bereits Kinder durch Schaffung eines entsprechenden Umfelds an ihrer Sozialisation mit, was Solms als "Multiplier Effect« bezeichnet: "although the innate differences might be tiny to begin with, they rapidly self-propagate and expand. $\ll^{16}$

Zum innerhalb der Neurowissenschaften selbst geführten Diskurs über die Natur des Menschen gehört auch, dass Neurowissenschaftler/-innen selbst die Frage stellen, ob Geschlecht im Sinne von Männlichkeit/Weiblichkeit überhaupt etwas ist, das zur biologischen Natur des Menschen gehört - oder ob diese Kategorien nicht vielmehr kultureller als biologischer Art sind. Diese Kritik bezieht sich sowohl auf methodische Probleme wie Versuchsaufbauten als auch auf das erkenntnistheoretische Problem des Voraussetzens von Kategorien und Konzeptionen - insbesondere der Gender Binary. ${ }^{17}$

\subsection{Neuroenhancement}

Mit dem Begriff Neuroenhancement wird die Einnahme von pharmakologischen Substanzen bezeichnet, die im Gegenüber zur therapeutisch eingesetzten Einnahme nicht medizinisch indiziert ist. Das Ziel liegt also nicht in der Linderung von Leid oder der Heilung von Krankheiten, sondern in der Steigerung der "Fähigkeiten des Menschen über die biostatistisch zu ermittelnde Schwelle arttypi-

\footnotetext{
${ }^{15}$ Swaab/Castellanos-Cruz/Bao, Brain and Gender (s. Anm. 13), 36.

${ }^{16}$ Solms, Biological Foundations (s. Anm. 12), 20; vgl. 19-20.

17 Seth Watt/Gillian Einstein, Beyond the Binary. The Corporeal Lives of Trans Individuals, in:Transsexualität in Theologie und Neurowissenschaften (s. Anm. 12), 55-74. Vgl. dazu auch Laura F. Mega, Wie Gender (auch) im Labor konstruiert und naturalisiert wird: Ein Fallbeispiel, in: Die Naturalisierung des Geschlechts. Zur Beharrlichkeit der Zweigeschlechtlichkeit, hg. v. Gero Bauer/Regina Ammicht Quinn/Ingrid Hotz-Davies, Bielefeld 2018, 43-57.
} 
scher Normalität hinaus « ${ }^{18}$. Gängige Beispiele für eine solche Praxis ist die Einnahme von aufmerksamkeitssteigernden Substanzen zur Leistungsverbesserung, etwa in anspruchsvollen familiären oder beruflichen Situationen, oder auch die Einnahme von stimmungsaufhellenden Präparaten durch nicht depressive Menschen. ${ }^{19}$

Gegen derartige Praktiken sind vielfache Einwände vorgebracht worden. ${ }^{20}$ In diesem Kontext wird die Frage gestellt, ob pharmakologische Eingriffe in das gesunde menschliche Gehirn unnatürlich sind und einen Eingriff in die menschliche Natur darstellen. Befürworter/-innen des Neuroenhancements wollen einen prinzipiellen Unterschied zwischen pharmakologischen und nichtpharmakologischen (z.B. Meditation) Selbst-Einflussnahmen nicht gelten lassen und hinterfragen, warum gerade diese Praktiken als unnatürlich bezeichnet und damit delegitimiert werden: "As for an appeal to the snaturak, the lives of almost all living humans are deeply unnatural $[\ldots]$. Given the many cognitive-enhancing tools we accept already, from writing to laptop computers, why draw the line here and say, thus far but no further? ${ }^{21}$ Darin spricht sich ein Zweites aus: Selbst wenn pharmakologische Eingriffe des Menschen unnatürlich wären, etwa weil es dadurch zu einer tatsächlichen Überschreitung der menschlichen Natur kommen könnte:Warum sollte diese Natur unbedingt schützenswert sein, wo doch der Mensch sonst überall

${ }^{18}$ Franz-Josef Bormann, Die Natur des Menschen als Grundlage der Moral? Zur Relevanz des Naturbegriffs für die Bio- und Neuroethik, in: Die "Natur des Menschen" in Neurowissenschaft und Neuroethik, hg. v. Jens Clausen/Oliver Müller/Giovanni Maio, Würzburg 2008, 13-36, hier 28.

${ }^{19}$ Diese dichotome Gegenüberstellung von sgesunden snormalen und sunnormalen Hirnfunktionen ist selbst Gegenstand von Kritik, etwa in Debatten um die Deutung von Autismus. Die Beschreibung von Autismus als Krankheit wird teilweise als unzulässige Pathologisierung empfunden und demgegenüber gefordert, Autismus als einen eigenständigen Weltzugang zu verstehen. Diese Forderung ist mit dem Stichwort "Neurodiversität " verbunden; Judy Singer, Why Can'tYou Be Normal for Once in Your Life? From a "Problem with no Name« to the Emergence of a New Category of Difference, in: Disability Discourse, hg. v. Mairian Corker/Sally French, Buckingham 1999, 59-67. Vgl. auch Ludger Tebartz van Elst, Disease, Human Norm, and Human Diversity in Neuropsychiatry, in: Philosophy, Theology and the Sciences 4 (2017), 143-159.

${ }^{20}$ Eine Übersicht über verschiedene Einwände gegen Neuroenhancement bieten Thorsten Galert u.a., Das optimierte Gehirn, in: Gehirn\&Geist 11 (2009) MEMORANDUM Neuro-Enhancement, 1-12, URL: https://www.spektrum.de/fm/976/ Gehirn_und_Geist_Memorandum.pdf (17.01.2019) - freilich mit dem Interesse, die Einwände zu widerlegen.

${ }^{21}$ Henry Greely u.a., Towards Responsible Use of Cognitive-enhancing Drugs by the Healthy, in: Nature 456 (2008), 702-705, hier 703, URL: https://www.nature. com/articles/456702a (17.01.2019). 
die Natur in seinem Interesse verändert?22 Und noch stärker: Gehört die Selbstgestaltung des Menschen im Sinne einer Optimierung nicht gerade unabdingbar zur Natur des Menschen? ${ }^{23}$ Ist "Natur" gar ein völlig ungeeignetes Kriterium zur Bewertung von Neuroenhancement?

Interessanterweise scheinen sich an dieser Stelle die Rollen umzukehren: Während in Debatten um das So-Sein des Menschen etwa in Bezug auf seine Freiheit oder seine Geschlechtlichkeit von neurowissenschaftlicher Seite klare Aussagen über die Natur des Menschen zu erwarten sind, bleibt die Natur des Menschen an dieser Stelle weniger klar umrissen. Die Neurowissenschaftler/-innen scheinen den Spiess geradezu umzukehren, indem sie ihren (geisteswissenschaftlichen) Kritiker/-innen den Vorwurf machen, mit willkürlichen und unterreflektiert Naturbegriffen zu operieren. ${ }^{24}$

\section{Ausblick}

Theolog/-innen haben angesichts der neurowissenschaftlichen Vermessung des Menschen und des damit einhergehenden Anspruchs, nun den Menschen voll und ganz erklären zu können, bereits auf die theologische Notwendigkeit hingewiesen, den Menschen als nicht vollständig beschreibbar zu verstehen. Christina Aus der Au macht auf die kritisch zu beobachtende Tendenz aufmerksam, dass neurowissenschaftliche Theorien beanspruchen, eine theory of everything darzustellen: ${ }^{25}$ "Was die Neurowissenschaften in letzter Konsequenz $\mathrm{zu}$ liefern beanspruchen [...], ist die letztgültige Beschreibung des

\footnotetext{
${ }^{22}$ Galert, Das optimierte Gehirn (s. Anm. 20), 3. Die Autorinnen und Autoren verweisen allerdings auch auf die Gefahren von pharmakologischen Enhancements, insofern Eingriffe in die Natur aufgrund des begrenzten Wissens über sie immer mit Risiken behaftet sind und deshalb mit Sorgfalt erfolgen müssen.

${ }^{23}$ Ebd., 11.

${ }^{24}$ Auf einen Mangel an Literatur zu Natürlichkeit als Argument im Hinblick auf Enhancement kann dieserVorwurf allerdings schwerlich beruhen. Einen guten Einstieg in die Debatten bietet Dietmar Hübner, Kultürlichkeit statt Natürlichkeit. Ein vernachlässigtes Argument in der bioethischen Debatte um Enhancement und Anthropotechnik, in: Jahrbuch für Wissenschaft und Ethik 19 (2015), 25-58.

${ }^{25}$ Christina Aus der Au, Wider die Beschreibbarkeit des Menschen, in: Theologie und Naturwissenschaften. Eine interdisziplinäre Werkstatt, hg. v. Frank Vogelsang u.a., Bonn 2006, 67-79, hier 69. Dies schliesst ein, eine Theorie der Theologie zu entwickeln. Noch deutlicher als in der neurowissenschaftlichen Forschung ist dies in der kognitionspsychologischen Religionsforschung; vgl. z.B. D. Jason Slone, Theological Incorrectness. Why Religious People Believe What They Shouldn't, New York/Oxford 2004.
} 
Menschen, welche nicht nur seine körperlichen Fähigkeiten und Eigenschaften umfasst, sondern auch sein Fühlen, sein Denken, sein Handeln. ${ }^{26}$ Diese Vorbehalte sind keineswegs überholt. Zugleich gebietet schon die eingangs erwähnte gesellschaftliche Relevanz neurowissenschaftlicher Forschung - und damit ihre Bedeutung für gegenwärtige Wirklichkeitsverständnisse -, ihren Beitrag zu Debatten über die Natur des Menschen genauer in den Blick zu nehmen, ob man sich ihren Thesen nun anschliessen möchte oder nicht. Dirk Evers' Charakterisierung der Aufgabe der Verhältnisbestimmung von Naturwissenschaften und Weltanschauung erscheint auch für die Verhältnisbestimmung von Theologie und Neurowissenschaften treffend zu sein:

"Wie können empirische Einzelerkenntnisse, schöpferische Theoriekonstruktionen und wissenstheoretisch reflektierte Voraussetzungen so ins Verhältnis gesetzt werden, dass das naturwiss[enschaftlich] gewonnene Bild der Welt nicht zu einer Weltanschauung mit Totalitätsanspruch wird, zugleich aber die Erklärungskompetenz der N[aturwissenschaften] angemessen zur Geltung kommt? «77

Wie kann das gelingen? Ich möchte mit drei Hinweisen schliessen, die mir für die theologische Forschung im Grenzbereich zu den Neurowissenschaften wichtig erscheinen:

Erstens sind Neurowissenschaften pluraler, als sie auf den ersten Blick erscheinen. Natürlich lassen sich provokante Thesen und Spitzensätze besser vermitteln als differenziertere Betrachtungen, weshalb manche Forscher/-innen den (gesellschaftlichen) Diskurs stärker prägen als andere. Es ist dabei auch für die Theologin leicht, derVersuchung zu erliegen, besonders diese Entwürfe ins Zentrum zu rücken. Gleichzeitig sollte dies nicht dazu führen, Neurowissenschaften ausschliesslich von diesen steilen Positionen her wahrzunehmen. Gerade angesichts von Entwicklungen innerhalb der Neurowissenschaften, die eine deutlicheVorsicht gegenüber den vormals formulierten wissenschaftstheoretischen und anthropologischen Erklärungsansprüchen formulieren, kann nach neuen Anknüpfungspunkten gesucht werden. Für die Theologie folgt daraus, dass sie sich darum bemühen muss, neurowissenschaftliche Theorien so präzise wie möglich wahrzunehmen und gerade auch an die vermeintlichen Ränder zu schauen.

\footnotetext{
${ }^{26}$ Aus der Au, Beschreibbarkeit (s. Anm. 25), 69.

${ }^{27}$ Dirk Evers, Art. Naturwissenschaft IV. Naturwissenschaft und Weltanschauung, in: ${ }^{4}$ R GG, Bd. 6, Tübingen 2003, 149.
} 
Es ist zweitens nicht von der Hand zu weisen, dass Verkürzungen und normative Aufladungen des Naturbegriffs kein Monopol der Neuro- bzw. Naturwissenschaften sind. Der Versuchung, die vermeintlich unveränderliche und unantastbare Natur des Menschen als unreflektiertes Autoritätsargument zu nutzen, können auch Geisteswissenschaftler/-innen erliegen. Mit Blick auf neuroethische Fragestellungen ist deshalb ein positiv gefüllter Naturbegriff ins Gespräch zu bringen.

Drittens erscheint mir in der zunehmenden Interdisziplinarität naturwissenschaftlicher Forschung eine wichtige Herausforderung für die Rezeption in der Theologie zu liegen. So urteilt etwa der Evolutionsbiologe Michael Tomasello, dass wahrhaft innovative Forschungsarbeit zumeist interdisziplinär angelegt ist, sowohl hinsichtlich der bearbeiteten Fragestellung als auch der Methoden - Wissenschaft passt (nicht mehr) in einfache disziplinäre Schubfächer. ${ }^{28}$ Aktuell wichtige Forschungszweige wie etwa die Kognitionswissenschaften (cognitive science) stellen sich von Anfang an als interdisziplinäres Unterfangen dar. ${ }^{29}$ Eben dies gilt auch für neurowissenschaftliche Forschung. Wer ist eigentlich der Gesprächspartner, wenn Theolog/-innen Ergebnisse »der Neurowissenschaften« rezipieren wollen? Schreibt da ein Psychologe, eine Philosophin, ein Biologe, eine Computerwissenschaftlerin? Wichtig ist auch hier, genau hinzuschauen: welche Methoden, welcher Anspruch, welche Intentionen, welche Verbindungslinien $\mathrm{zu}$ anderen Wissenschaften gibt es? Vielleicht ist die Theologie durch ihre intradisziplinäre Zusammensetzung als praktische Wissenschaft für eben diese Gesprächssituation schon bestens vorbereitet.

- Laura-Christin Krannich ist Doktorandin am Lehrstuhl für Dogmatik und Religionswissenschaft der Martin-Luther-Universität Halle-Wittenberg. Ihre Arbeitsschwerpunkte umfassen kognitionswissenschaftlichen Religionsforschung (Cognitive Science of Religion) sowie Hermeneutische Theologie und Geschlechterforschung.

\footnotetext{
${ }^{28}$ Michael Tomasello, Die kulturelle Entwicklung des menschlichen Denkens. Zur Evolution der Kognition, übers. v. Jürgen Schröder, Frankfurt a.M. 2002, 7-10.

${ }^{29}$ Zur Disziplingeschichte der Kognitionswissenschaften vgl. George A. Miller, The Cognitive Revolution. A Historical Perspective, in:TRENDS in Cognitive Sciences 7 (2003), 141-143, mit einer bildlichen Darstellung der interdisziplinären Zusammensetzung (143).
} 